\title{
Formulation and Evaluation of Orodispersible Tablet of Atorvastatin Calcium by Using Hibiscus rosa sinesis Mucilage as Natural Superdisintegrant
}

\author{
Gupta Ashish*, Bhadoria Juhi, Darwhekar G.N. \\ Acropolis Institute of Pharmaceutical Education and Research, Indore, Indore Dewas Bypass Road,Manglia Square, Indore (M.P.) 453771
}

\begin{abstract}
Orodispersible tablets (ODTs), also known as fast melt, quick melts, fast disintegrating have the unique property of disintegrating in the mouth in seconds without chewing and the need of water. Oral bioavailability of Atorvastatin Calcium is low (14\%) and shows extensive intestinal clearance and first-pass metabolism, which is the main cause for the low systemic availability. In the present work, orodispersible tablets of Atorvastatin calcium were prepared by direct compression method using Hibiscus rosa sinesis mucilage as natural superdisintegrant with a view to enhance patient compliance and to avoid hepatic first pass metabolism and to improve its bioavailability. The prepared batches of tablets were evaluated for hardness, friability, drug content uniformity, wetting time, water-absorption ratio and in-vitro dispersion time. Short-term stability studies on the promising formulation indicated that there are no significant changes in drug content and in vitro dispersion time.
\end{abstract}

Keywords: Orodispersible tablet, Atorvastatin Calcium, lipid-lowering agent, Superdisintegrant, Hibiscus Rosa Sinensis, Bioavailability, solubility.

\section{Article Info: Received 10 Sep 2019; Review Completed 18 Oct 2019; Accepted 28 Oct 2019; Available online 15 Nov 2019}

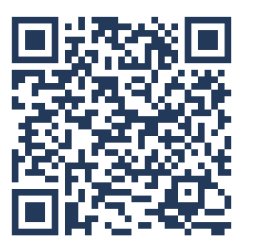

\section{Cite this article as:}

Gupta A, Bhadoria J, Darwhekar GN, Formulation and Evaluation of Orodispersible Tablet of Atorvastatin Calcium by Using Hibiscus rosa sinesis Mucilage as Natural Superdisintegrant, Journal of Drug Delivery and Therapeutics. 2019; 9(6):90-94 http://dx.doi.org/10.22270/jddt.v9i6.3677

Gupta Ashish, Acropolis Institute of Pharmaceutical Education and Research, Indore, Indore Dewas Bypass Road,Manglia Square, Indore (M.P.) 453771

\section{INTRODUCTION}

Oral delivery is current standard in the pharmaceutical industry wherever it is regarded as the safest, most suitable and most economical method of drug delivery. The oral cavity is an attractive site for the administration of drugs because of ease of administration.

Oro-dispersible drug delivery system are Novel Drug Delivery techniques that make the tablets disintegrate in the mouth without chewing and water, and immediate release and enhanced bioavailability, with better patient compliance.

Recently, the European Pharmacopeia adopted the term orodispersible tablet for a tablet that disperses or disintegrates within a minute or second in the mouth before swallowing.

United States Food and Drug Administration (FDA) defined Oro-dispersible tablet as "a solid dosage form containing medicinal substances or active ingredient which disintegrate or dissolve rapidly within seconds when placed upon the tongue.
Oro-dispersible tablets have a quick dissolution and rapid absorption which provide rapid onset of action. Moreover, drug candidates that undergo pregastric absorption when formulated as ODTs may oral bioavailability of drug is enhanced by avoiding the hepatic first pass metabolism. It provides good stability, accurate dosing, easy of manufacturing. Oro-dispersible tablets are made by a direct compression method using super Disintegrate as an important component.

\section{MATERIAL AND METHODS}

\section{Materials}

Atorvastatin calcium was obtained as a gift sample from Yarrowchem Products (Mumbai). Beta-cyclodextrin was obtained as a gift sample from Alkem laboratories Ltd (Mumbai). Microcrystalline Cellulose, Mannitol, Magnesium Stearate, Talc, Ethanol, Methanol and Silica gel-G, Lobachem Pvt. Ltd. Aspartame, Potassium Dihydrogen Phosphate, Sodium hydroxide and Hydrochloric acid were purchased from Lobachem and Merck Pvt Ltd. 


\section{Methods}

\section{Drug Characterization}

\section{Melting point determination}

Capillary tube was taken and one end was sealed by heating. Capillary tube was filled with drug powder upto 2-3mm high. The capillary tube was putted inside melting point apparatus and temperature was increased slowly. The temperature was noted when the drug gets starts melting and again noted when drugs completely melted.

\section{UV Spectroscopy}

$50 \mathrm{mg}$ of drug was weighed and was dissolved in $50 \mathrm{ml}$ of methanol $(1 \mathrm{mg} / \mathrm{ml}) .10 \mathrm{ml}$ of this solution was withdrawn and volume was made up to $100 \mathrm{ml}$. Appropriate dilutions were made with methanol to give concentration of $10 \mu \mathrm{g} / \mathrm{ml}$, scanned in UV range from $200-400 \mathrm{~nm}$, which could be utilized for analysis and spectrum was recorded.

\section{Calibration curve}

Accurately weighed $50 \mathrm{mg}$ of Atorvastatin Calcium was transferred into a $500 \mathrm{ml}$ volumetric flask \& dissolved in phosphate buffer 6.8. Then sonicated for 15 minute and the volume was made up with phosphate buffer $\mathrm{pH} 6.8$ to obtain a $100 \mu \mathrm{g} / \mathrm{ml}$ stock solution of Atorvastatin Calcium.

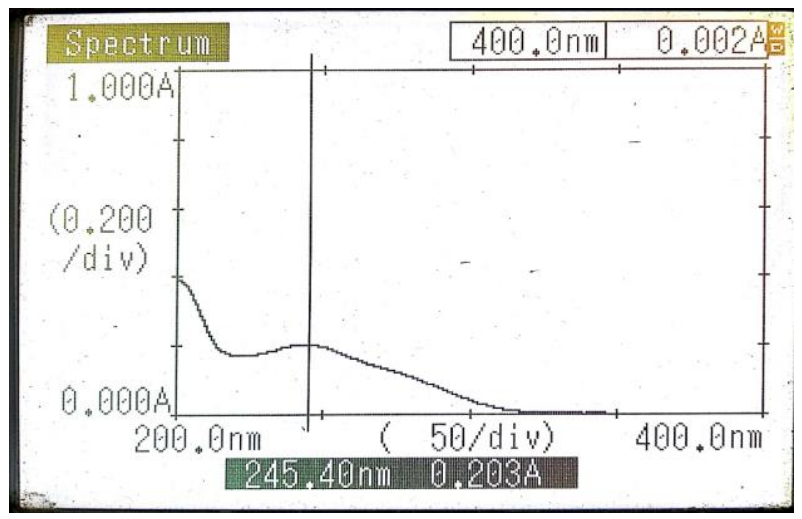

Fig. No. 1 Lambda Max of Atorvastatin Calcium

\section{Solubility studies}

An excess amount of prepared Atorvastatin- $\beta$-cyclodextrin inclusion complex at different concentration $(1: 3,1: 4,1: 5)$ were separately dissolved in $5 \mathrm{ml}$ phosphate buffer $\mathrm{pH} 6.8$ in vials and sealed properly and stirred continuously. The process was repeated until saturation solubility of inclusion complex. The solution was kept for 24 hours at room temperature. Then solution was filtered. The adequately diluted with phosphate buffer $\mathrm{pH}$ 6.8. Then solution was analyzed by using UV-visible spectrophotometer at $245.40 \mathrm{~nm}$.

Table no.1: Solubility data of Atorvastatin Calcium in different mediums:

\begin{tabular}{|c|c|c|c|}
\hline S.NO. & Solvent & $\begin{array}{c}\text { Solubility (mg/ml) } \\
\text { Mean } \pm \text { SD (n=3) }\end{array}$ & Inference \\
\hline 1 & Methanol & $30.106 \pm 2.186$ & Soluble \\
\hline 2 & Phosphate buffer pH 6.8 & $0.184 \pm 0.001$ & Very Slightly soluble \\
\hline 3 & pH 1.2 HCl buffer & $0.108 \pm 0.007$ & Very Slightly soluble \\
\hline 4. & Water & $0.578 \pm 0.010$ & Slightly soluble \\
\hline
\end{tabular}

\section{Formulation}

\section{- Solubility enhancement by Inclusion Complex}

An excess amount of prepared Atorvastatin- $\beta$-cyclodextrin inclusion complex at different concentration $(1: 3,1: 4,1: 5)$ were separately dissolved in $5 \mathrm{ml}$ phosphate buffer $\mathrm{pH} 6.8$ in vials and sealed properly and stirred continuously. The process was repeated until saturation solubility of inclusion complex. The solution was kept for 24 hours at room temperature then solution was filtered. The solubility was determined by using UV- Spectroscopy.

Table no. 2

\begin{tabular}{|c|c|c|c|}
\hline S.No. & Phosphate buffer pH 6.8 & $\begin{array}{c}\text { Solubility } \mathbf{~} \mathbf{m g} / \mathbf{m l}) \\
\text { Mean } \pm \text { SD }(\mathbf{n = 3})\end{array}$ & Inference \\
\hline 1 & Pure drug & $0.184 \pm 0.001$ & Very Slightly Soluble \\
\hline 2 & Drug: $\beta$-CD $(1: 3)$ & $0.471 \pm 0.004$ & Slightly Soluble \\
\hline 3 & Drug: $\beta-C D(1: 4)$ & $0.694 \pm 0.008$ & Slightly Soluble \\
\hline 4 & Drug: $\beta-C D(1: 5)$ & $0.840 \pm 0.012$ & Slightly Soluble \\
\hline
\end{tabular}

\section{- Experimental design}

A two factor three level factorial design $\left(3^{2}\right)$ was used for the formulation optimization of orodispersible tablet of Atorvastatin and experimental trials are performed at all 9 possible formulation. In which the amount of $\beta$-cyclodextrin
(X1) and Hibiscus Rosa- Senesis mucilage (X2) were selected as independent variables (factor) varied at three different level: low(-1), medium(0), and high(+1) levels. 
Table no. 3

\begin{tabular}{|c|c|c|c|c|c|c|c|c|c|c|}
\hline $\begin{array}{l}\text { S. } \\
\text { No }\end{array}$ & Name of ingredients & $\begin{array}{c}\text { F1 } \\
\text { (mg) }\end{array}$ & $\begin{array}{c}\text { F2 } \\
(\mathrm{mg})\end{array}$ & $\begin{array}{c}\text { F3 } \\
\text { (mg) }\end{array}$ & $\begin{array}{c}\text { F4 } \\
\text { (mg) }\end{array}$ & $\begin{array}{c}\text { F5 } \\
\text { (mg) }\end{array}$ & $\begin{array}{c}\text { F6 } \\
(\mathrm{mg})\end{array}$ & $\begin{array}{c}\text { F7 } \\
\text { (mg) }\end{array}$ & $\begin{array}{c}\text { F8 } \\
(\mathrm{mg})\end{array}$ & $\begin{array}{c}\text { F9 } \\
\text { (mg) }\end{array}$ \\
\hline 1. & Drug (Atorvastatin) & 10 & 10 & 10 & 10 & 10 & 10 & 10 & 10 & 10 \\
\hline 2. & $\beta$-cyclodextrin & 30 & 30 & 30 & 40 & 40 & 40 & 50 & 50 & 50 \\
\hline 3. & $\begin{array}{l}\text { Hibiscus Rosa- Senesis } \\
\text { Mucilage }\end{array}$ & 4 & 6 & 8 & 10 & 12 & 14 & 16 & 18 & 20 \\
\hline 4. & Microcrystalline cellulose & 120 & 120 & 120 & 120 & 120 & 120 & 120 & 120 & 120 \\
\hline 5. & Mannitol & 40 & 40 & 40 & 40 & 40 & 40 & 40 & 40 & 40 \\
\hline 6. & Aspartame & 7.5 & 7.5 & 7.5 & 7.5 & 7.5 & 7.5 & 7.5 & 7.5 & 7.5 \\
\hline 7. & Magnessium Stearate & 4.5 & 4.5 & 4.5 & 4.5 & 4.5 & 4.5 & 4.5 & 4.5 & 4.5 \\
\hline 8. & Talc & 4.5 & 4.5 & 4.5 & 4.5 & 4.5 & 4.5 & 4.5 & 4.5 & 4.5 \\
\hline
\end{tabular}

\section{Preparation of Atorvastatin Calcium Fast Disssolving tablet by Direct compression method}

Orodispersible tablet of Atorvastatin were prepared by direct compression method. Weighed all the ingredients accurately according to the table no.3. All the ingredients except Talc, Magnessium sterate were mixed step by step and trituration was continued for 15 minute, and passed through sieve no. \#60. Subsequently talc, magnesium stearate mixed at last \& again mixed. The powder was compressed using multistation tablet punching machine (Aidmach Pvt. Ltd.) with $8 \mathrm{~mm}$ flat punch, B-tooling and corresponding dies.

\section{Evaluation of Tablet}

\section{- Weight variation}

Randomly, twenty tablets were selected after compression and the mean weight was determined. None of the tablets deviated from the average weight by more than $\pm 7.5 \%$ (USPXX).

\section{- Thickness}

Thickness of tablet was determined by using vernier calliper (Mitutoya, Model CD-6 CS, Japan).

\section{- Hardness}

The crushing strength of the tablets was measured using a Monsanto hardness tester (Sheetal Scientific Industries, Mumbai, India). Three tablets from each formulation batch were tested randomly and the average reading noted.

\section{- Friability}

Twenty tablets were weighed and placed in a Roche friabilator (Electrolab, India). Twenty reweighed tablets were rotated at $25 \mathrm{rpm}$ for $4 \mathrm{~min}$. The tablets were then dedusted and reweighed and the percentage of weight loss was calculated. The percentage friability of the tablets were measured as per the following formula,

Percentage friability $=$ Initial weight-Final weight $\mathrm{x} 100$

$$
\text { Initial weight }
$$

\section{- Wetting time}

A piece of circular tissue paper $(8 \mathrm{~cm})$ folded twice was placed in a Petri dish (Internal Diameter $=9 \mathrm{~cm}$ ) containing $10 \mathrm{ml}$ of buffer solution simulating saliva $\mathrm{pH}$ 6.8. A tablet was placed on the paper and the time taken for complete wetting was noted. Three tablets from each formulation were randomly selected and the average wetting time was noted. The results are tabulated in Table 6 .

\section{- Water absorption ratio}

A piece of tissue paper folded twice was placed in a small petri dish containing $6 \mathrm{ml}$ of water. A tablet was put on the tissue paper and allowed to completely wet. The wetted tablet was the reweighed. Water absorption ratio, $\mathrm{R}$ was determined using following equation-

$$
\mathrm{R}=100 \times \mathrm{Wa}-\mathrm{Wb} / \mathrm{Wa}
$$

Where, $\mathrm{Wa}=$ Weight of tablet after water absorption $\mathrm{Wb}=$ Weight of wetted tablet before water absorption

\section{- Disintegration Time}

One tablet was introduced into each tube and disc was added to each tube. The assembly was introduced in the beaker containing purified water. The apparatus operated until the tablet completely disintegrate. The time was noted down until the tablets completely disintegrate. The assembly was removed from water.

\section{- Dissolution Study}

In vitro release of Atorvastatin calcium from tablets was monitored by using $900 \mathrm{ml}$ of simulated intestinal fluid, SIF (USP phosphate buffer solution, $\mathrm{pH} 6.8$ ) at $37 \pm 0.5^{\circ} \mathrm{C}$ and 50 rpm using programmable dissolution tester [Paddle type, model TDT-08L, Electrolab, (USP), India]. $5 \mathrm{ml}$ Aliquots were withdrawn at one minute time intervals and were replenished immediately with the same volume of fresh buffer medium. Aliquots, following suitable dilutions, were assayed spectrophotometrically (UV-1700, Shimadzu, Japan) at $245.5 \mathrm{~nm}$.

\section{RESULT AND DISCUSSION}

Mouth dissolving drug delivery is rapidly gaining acceptance as an important drug delivery technology. In such drug delivery, different dosage forms disintegrate rapidly in the patient's mouth within a minute and can be gulped easily without need of water. This rapid disintegration can be achieved by use of high levels of disintegrate and/or effervescent agents along with water soluble diluents. Hence, it offers increased patient compliance and convenience. orodispersible tablet of Atorvastatin calcium was formulated. Total nine batches were prepared for orodispersible formulation. All the formulations were subjected to evaluation, Tablet weight varied from 110 to $120 \mathrm{mg}$, and thickness 3 to $4.1 \mathrm{~mm}$. All the tablets exhibited friability values between 0.5 to 0.8 , all tablets disintegrated in less than 1 minute. The drug released at the time interval of 30 minutes upto $97.5 \%$ of batch F9. 
Table no. 4 Evaluation of Flow properties of powder (Drug excipient mixture)

\begin{tabular}{|c|c|c|c|c|c|}
\hline Formulation & $\begin{array}{c}\text { Bulk density } \\
\text { (gm/ml) } \\
(\mathrm{n}=3) \\
\text { Mean } \pm \text { SD }\end{array}$ & $\begin{array}{c}\text { Tapped density } \\
\text { (gm/ml) } \\
\text { Mean } \pm \text { SD }\end{array}$ & $\begin{array}{c}\text { Carr's index (\%) } \\
(n=3) \\
\text { Mean } \pm \text { SD }\end{array}$ & $\begin{array}{c}\text { Angle of repose } \\
\text { (o) }(n=3) \\
\text { Mean } \pm \text { SD }\end{array}$ & $\begin{array}{c}\text { Hausner's } \\
\text { ratio } \\
(n=3) \\
\text { Mean } \pm S D\end{array}$ \\
\hline MD1 & $0.314 \pm 0.004$ & $0.368 \pm 0.004$ & $14.635 \pm 1.503$ & $25.406 \pm 0.374$ & $1.170 \pm 0.026$ \\
\hline MD2 & $0.299 \pm 0.002$ & $0.358 \pm 0.003$ & $14.634 \pm 1.004$ & $29.333 \pm 1.106$ & $1.166 \pm 0.015$ \\
\hline MD3 & $0.288 \pm 0.002$ & $0.345 \pm 0.003$ & $16.323 \pm 1.047$ & $27.606 \pm 0.525$ & $1.186 \pm 0.015$ \\
\hline MD4 & $0.332 \pm 0.003$ & $0.385 \pm 0.006$ & $13.907 \pm 0.852$ & $26.966 \pm 0.450$ & $1.16 \pm 0.01$ \\
\hline MD5 & $0.307 \pm 0.003$ & $0.363 \pm 0.002$ & $15.260 \pm 1.695$ & $25.59 \pm 0.213$ & $1.176 \pm 0.020$ \\
\hline MD6 & $0.293 \pm 0.003$ & $0.355 \pm 0.003$ & $17.360 \pm 1.663$ & $27.4 \pm 0.500$ & $1.206 \pm 0.025$ \\
\hline MD7 & $0.344 \pm 0.003$ & $0.402 \pm 0.004$ & $14.414 \pm 0.402$ & $26.74 \pm 0.767$ & $1.166 \pm 0.005$ \\
\hline MD8 & $0.326 \pm 0.003$ & $0.376 \pm 0.003$ & $13.354 \pm 1.494$ & $30.6 \pm 0.888$ & $1.15 \pm 0.02$ \\
\hline MD9 & $0.298 \pm 0.002$ & $0.330 \pm 0.001$ & $9.787 \pm 0.459$ & $26.633 \pm 0.709$ & $1.103 \pm 0.005$ \\
\hline
\end{tabular}

Table no. 5 Determination of physicochemical properties of orodispersible tablet

\begin{tabular}{|c|c|c|c|}
\hline Formulation & $\begin{array}{c}\text { Weight variation } \\
\text { (mg) }(n=3) \\
\text { Mean } \pm \text { SD }\end{array}$ & $\begin{array}{c}\text { Hardness } \\
\left(\mathrm{Kg} / \mathrm{cm}^{2}\right) \\
(\mathrm{n}=3) \\
\text { Mean } \pm \text { SD }\end{array}$ & $\begin{array}{c}\text { Friability } \\
(\%) \\
(n=3) \\
\text { Mean } \pm \text { SD }\end{array}$ \\
\hline MD1 & $210.16 \pm 0.378$ & $2.6 \pm 0.264$ & $0.460 \pm 0.027$ \\
\hline MD2 & $211.75 \pm 0.312$ & $2.4 \pm 0.173$ & $0.750 \pm 0.047$ \\
\hline MD3 & $214 \pm 0.938$ & $3.0 \pm 0.057$ & $0.460 \pm 0.045$ \\
\hline MD4 & $223 \pm 0.301$ & $2.9 \pm 0.152$ & $0.672 \pm 0.045$ \\
\hline MD5 & $232 \pm 2.13$ & $3.0 \pm 0.1$ & $0.346 \pm 0.043$ \\
\hline MD6 & $233 \pm 0.28$ & $3.0 \pm 0.057$ & $0.343 \pm 0.043$ \\
\hline MD7 & $246.58 \pm 0.56$ & $3.0 \pm 0.057$ & $0.349 \pm 0.023$ \\
\hline MD8 & $247.83 \pm 1.05$ & $3.1 \pm 0.1$ & $0.361 \pm 0.040$ \\
\hline MD9 & $251.06 \pm 0.17$ & $3.1 \pm 0.057$ & $0.358 \pm 0.040$ \\
\hline
\end{tabular}

Table no. 6 Other Evaluation parameters

\begin{tabular}{|c|c|c|c|c|}
\hline Formulation & $\begin{array}{c}\text { Disintegration } \\
\text { Time (sec) } \\
(n=3) \\
\text { Mean } \pm \text { SD }\end{array}$ & $\begin{array}{c}\text { Drug Content } \\
(\%) \\
(\mathrm{n}=3) \\
\text { Mean } \pm \text { SD }\end{array}$ & $\begin{array}{c}\text { Wetting time } \\
(\mathrm{sec}) \\
(\mathrm{n}=3) \\
\text { Mean } \pm \text { SD }\end{array}$ & $\begin{array}{c}\text { Water absorption } \\
\text { Ratio }(\%)(n=3) \\
\text { Mean } \pm \text { SD }\end{array}$ \\
\hline MD1 & $42 \pm 1.05$ & $91.833 \pm 0.233$ & $32.7 \pm 0.590$ & $55.97 \pm 3.63$ \\
\hline MD2 & $38 \pm 1.86$ & $93.36 \pm 0.356$ & $31.37 \pm 0.580$ & $49.01 \pm 3.59$ \\
\hline MD3 & $37 \pm 1.93$ & $95.84 \pm 1.362$ & $30.05 \pm 0.040$ & $42.18 \pm 3.13$ \\
\hline MD4 & $35 \pm 1.28$ & $92.19 \pm 0.583$ & $32.38 \pm 0.540$ & $46.75 \pm 1.34$ \\
\hline MD5 & $30 \pm 1.25$ & $99.25 \pm 0.470$ & $29.71 \pm 0.546$ & $30.3 \pm 1.56$ \\
\hline MD6 & $33 \pm 1.36$ & $96.85 \pm 0.584$ & $31.06 \pm 0.015$ & $35.90 \pm 0.65$ \\
\hline MD7 & $45 \pm 1.68$ & $95.76 \pm 0.466$ & $33.38 \pm 0.580$ & $36.83 \pm 0.61$ \\
\hline MD8 & $36 \pm 1.26$ & $95.60 \pm 1.151$ & $34.69 \pm 0.534$ & $40.42 \pm 0.61$ \\
\hline MD9 & $39 \pm 1.48$ & $93.73 \pm 1.113$ & $35.06 \pm 0.015$ & $41.96 \pm 0.60$ \\
\hline
\end{tabular}

Table no. 7 Invitro drug release study

\begin{tabular}{|c|c|c|c|c|c|c|c|c|c|}
\hline \multirow{2}{*}{$\begin{array}{c}\text { Time } \\
\text { (in min }\end{array}$} & \multicolumn{9}{|c|}{ \% Cumulative drug Release (Mean士SD) $(n=3)$} \\
\hline & MD1 & MD2 & MD3 & MD4 & MD5 & MD6 & MD7 & MD8 & MD9 \\
\hline 0 & 0 & 0 & 0 & 0 & 0 & 0 & 0 & 0 & 0 \\
\hline 5 & $11.4 \pm 3.20$ & $\begin{array}{l}16.9 \pm \\
3.16\end{array}$ & $\begin{array}{l}21.84 \pm \\
4.35\end{array}$ & $\begin{array}{l}28.83 \pm \\
2.11\end{array}$ & $\begin{array}{l}27.44 \pm \\
2.41\end{array}$ & $\begin{array}{l}30.24 \pm \\
3.27\end{array}$ & $\begin{array}{l}21.15 \pm 3 . \\
21\end{array}$ & $\begin{array}{l}23.94 \pm \\
3.21\end{array}$ & $\begin{array}{l}21.84 \pm \\
2.44\end{array}$ \\
\hline 10 & $\begin{array}{l}214 \pm \\
1.19\end{array}$ & $\begin{array}{l}25.3 \pm \\
1.60\end{array}$ & $\begin{array}{l}37.2 \pm \\
2.11\end{array}$ & $\begin{array}{l}34.2 \pm \\
2.11\end{array}$ & $\begin{array}{l}35.1 \pm \\
2.07\end{array}$ & $\begin{array}{l}39.99 \pm \\
3.20\end{array}$ & $\begin{array}{l}28.83 \pm \\
2.11\end{array}$ & $\begin{array}{l}34.44 \pm \\
4.35\end{array}$ & $\begin{array}{l}28.14 \pm \\
3.20\end{array}$ \\
\hline 15 & $\begin{array}{l}34.41 \pm \\
3.16\end{array}$ & $\begin{array}{l}40.0 \pm \\
1.20\end{array}$ & $\begin{array}{l}46.29 \pm \\
2.44\end{array}$ & $\begin{array}{l}44.21 \pm \\
3.18\end{array}$ & $\begin{array}{l}46.98 \pm \\
3.21\end{array}$ & $\begin{array}{l}44.87 \pm \\
1.280\end{array}$ & $\begin{array}{l}41.14 \pm \\
2.07\end{array}$ & $\begin{array}{l}43.51 \pm \\
3.62\end{array}$ & $\begin{array}{l}33.71 \pm \\
3.16\end{array}$ \\
\hline 20 & $\begin{array}{l}44.2 \pm \\
4.37\end{array}$ & $\begin{array}{l}53.9 \pm \\
5.52\end{array}$ & $\begin{array}{l}57.45 \pm \\
6.41\end{array}$ & $\begin{array}{l}50.51 \pm \\
4.81\end{array}$ & $\begin{array}{l}56.07 \pm \\
2.07\end{array}$ & $\begin{array}{l}55.34 \pm 3.2 \\
2\end{array}$ & $\begin{array}{l}53.24 \pm \\
2.45\end{array}$ & $\begin{array}{l}56.76 \pm \\
1.95\end{array}$ & $\begin{array}{l}47 \pm \\
3.21\end{array}$ \\
\hline 25 & $\begin{array}{l}58.14 \pm \\
2.07\end{array}$ & $\begin{array}{l}67.9 \pm \\
5.25\end{array}$ & $\begin{array}{l}63.72 \pm \\
4.34\end{array}$ & $\begin{array}{l}67.92 \pm \\
3.20\end{array}$ & $\begin{array}{l}73.14 \pm \\
2.14\end{array}$ & $\begin{array}{l}62.24 \pm 1.9 \\
6\end{array}$ & $\begin{array}{l}75.59 \pm \\
3.21\end{array}$ & $\begin{array}{l}72.83 \pm \\
2.07\end{array}$ & $\begin{array}{l}68.61 \pm \\
2.11\end{array}$ \\
\hline 30 & $\begin{array}{l}90.94 \pm \\
3.17\end{array}$ & $\begin{array}{l}86.0 \pm 1.2 \\
4\end{array}$ & $\begin{array}{l}89.55 \pm \\
3.58\end{array}$ & $\begin{array}{l}88.13 \pm \\
3.19\end{array}$ & $\begin{array}{l}95.84 \pm \\
2.08\end{array}$ & $\begin{array}{l}84.66 \pm 3.2 \\
0\end{array}$ & $\begin{array}{l}86.76 \pm \\
1.24\end{array}$ & $\begin{array}{l}92.34 \pm \\
4.34\end{array}$ & $\begin{array}{l}93.72 \pm \\
2.09\end{array}$ \\
\hline
\end{tabular}




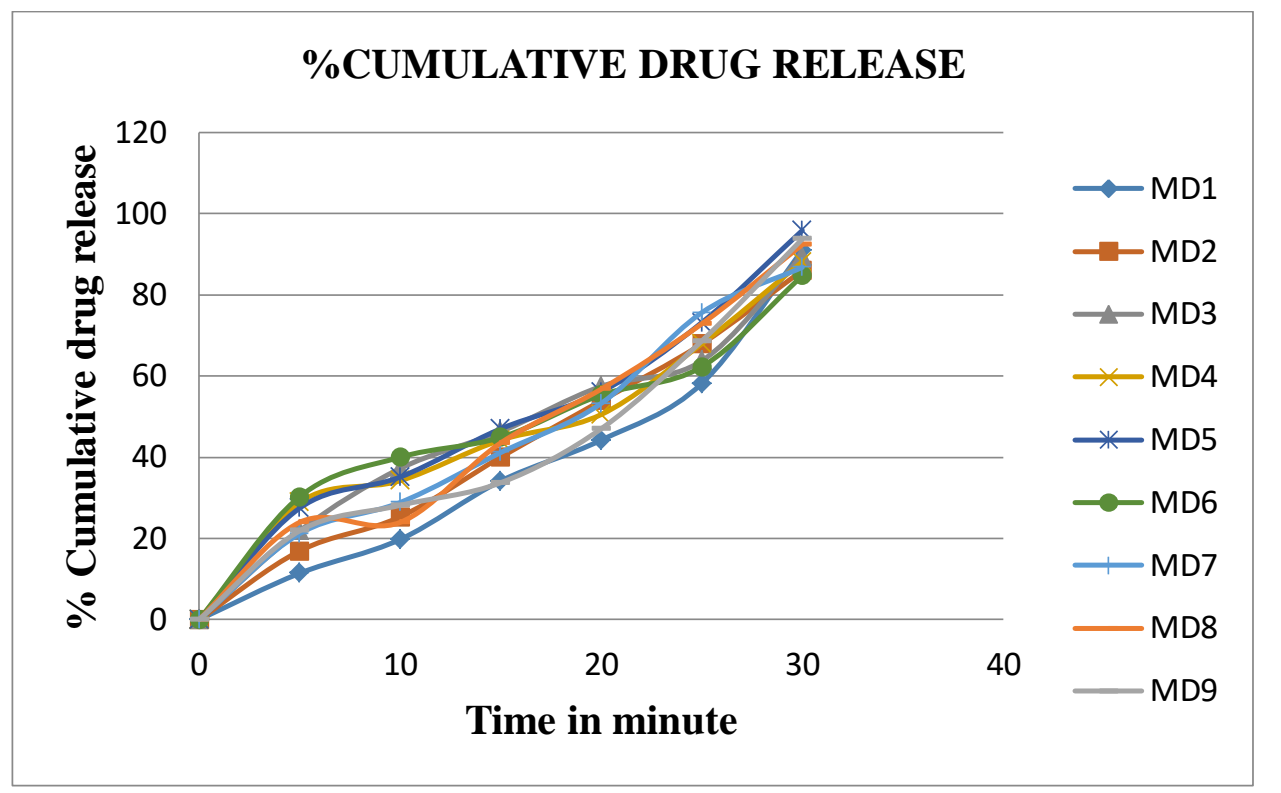

Fig no. 2 Invitro drug release of orodispersable tablet

Table no. 8 Stability studies

\begin{tabular}{|c|c|c|c|}
\hline S.No. & Parameter & After 30 days & Inference \\
\hline 1. & Weight variation & $229.33 \pm 0.574$ & Within Limit \\
\hline 2. & Hardness & $3.03 \pm 0.055$ & Within Limit \\
\hline 3. & Drug content & $99.36 \pm 0.568$ & Within Limit \\
\hline 4. & Wetting time & $27.66 \pm 0.364$ & Within Limit \\
\hline 5. & Water absorption ratio & $33.66 \pm 0.475$ & Within Limit \\
\hline 6. & Disintegration & $33.48 \pm 0.575$ & Within Limit \\
\hline
\end{tabular}

\section{CONCLUSION}

The present study was carried out to prove that Orodispersible tablet of Atorvastatin calcium can be formulated. The concept explains that formulated tablets avoids hepatic first pass metabolism and improves its bioavailability.

\section{ACKNOWLEDGMENT}

The authors are thankful to acropolis institute of pharmaceutical education and research, Indore for providing necessary facilities in the institute. Sincerely thanks to Yarrowchem Products and Alkem laboratories Ltd for providing drug and Beta-cyclodextrin respectively as gift sample.

\section{REFERENCES}

1. Gauri S, Kumar G. Fast Dissolving Drug Delivery and its Technologies. The Pharma Innovation, 2012; 1(2):34-39.

2. Mudgal, V. K., Sethi P., Kheri R., Saraogi G.K., Singhai A.K. Orally Disintegrating Tablets: A Review. International Research Journal of Pharmacy, 2011; 2(4):16 -22.

3. Mehta K., Garala K., Basu B., Bhalodia R., Joshi B., Charyulu N. An Emerging Trend in Oral Drug Delivery Technology: Rapid Disintegrating Tablets. Journal of Pharmaceutical Science and Technology, 2010; 2(10):318-329.

4. Shihora H., Panda S. Superdisintegrants, utility in Dosage Forms: A Quick Review. Journal of Pharmaceutical Science and Biosientipic Resarch, 2011; 1(3):148-153.

5. Sharma V., Arora V., Ray C. Use of Natural superdisintegrant in Mouth Dissolving Tablet an Emerging Trend. International Bulletin of Drug Research, 2010; 1(2):46-54.
6. Bala R., *Madaan R., Vibhu A. And Dr. Arora S. Isolation and Evaluation Of Hibiscus Rosa- Sinensis Leaf Mucilage As Superdisintegrant. European Journal of Pharmaceutical and Medical Research. ejpmr, 2016; 3(8):434-440

7. Carrier R.L., Miller L.A., Ahmed I. The utility of cyclodextrins for enhancing oral bioavailability. J Control Release, 2007; 123:78e99.

8. Singh A., Worku Z.A., Van den Mooter G. Oral formulation strategies to improve solubility of poorly water-soluble drugs Expert Opin Drug Delivery, 2011; 8:1361-1378.

9. Rowe R.C., Sheskey P.J. and Quinn M.E. Handbook of Pharmaceutical Excipients Sixth edition published. Published by the Pharmaceutical Press, 2009; 404-407.

10. Jadhav N.R., Kambar R.S. and Nadaf S.J. Research Article Dual Wavelength Spectrophotometric Method for Simultaneous Estimation of Atorvastatin Calcium and Felodipine from Tablet Dosage Form Hindawi Publishing Corporation Advances in Chemistry Volume, Article ID, 2014; 131974, 6.

11. Rao K.S. Enhancement of dissolution rate and bioavailability of Aceclofenac by Complexation with cyclodextrin Research Journal of Pharmaceutical and Chemical Science Oct-Dec, 2010; 1(4):142-152.

12. Stability testing of new drug substances and products, ICH Guidelines, 6 February 2003, Available at: https://www.ich.org/fileadmin/Public_Web_Site/ICH_Product s/Guidelines/Quality/Q1A_R2/Step4/Q1A_R2_Guideline.pdf.

13. Umekar M.J. Formulation and Characterization of lornoxicam fast dissolving tablet using natural superdisintegrants, International Journal of Research in Pharmaceutical and Biomedical Sciences, 2013; 2:459-64.

14. Goyani S.M., Shah P. Formulation and evaluation of orally disintegrating tablets of meclizine hydrochloride Int. Res. J. Pharm, 2012; 3(3):196-99. 